\title{
Apuntes sobre el paisaje social y natural
}

\begin{abstract}
Resumen
El artículo desarrolla, a partir del conocimiento del proyecto de Recuperación del Capital Físico y Social de las lomas de Pachacámac, la actitud de respeto hacia el paisaje social y la naturaleza que transmiten, tácitamente, las campañas de propaganda, la construcción de un marco teórico amplio y sólido que sustenten los proyectos relacionados al medio ambiente y la importancia de la enseńanza de la ecología en las universidades. En el texto se revalora a la población indígena, porque su presencia en el mundo ha permitido la conservación de muchos ecosistemas de enorme significación para la humanidad. Finalmente, se propone, al comunicador, la reflexión sobre su propio conocimiento, la urgencia de una educación masiva en temas ecológicos y la necesidad de legislar respecto al uso de los recursos naturales.
\end{abstract}

Palabras clave: Medio ambiente, ecología, marco teórico, recursos naturales y reflexión del comunicador.

\section{Notes on natural and social landscape}

\begin{abstract}
From the knowledge on the Physical and Social Capital Recovery of lomas de Pachacamac project, the present article develops the attitude of respect towards Nature implicitly transmitted through propaganda campaigns, a comprehensive and consistent theoretical framework building, supporting the projects related to the environment and the importance of ecology education in universities. In the present article, the indigenous population is revaluated, since their presence in the world has allowed the preservation of many ecosystems of great significance to humanity. Finally, the communicator is advised to reflect on his own knowledge, the emergency of mass education in ecological issues and the need to legislate regarding to the use of natural resources.

Mg. Gina Gogin Sias Magíster en Antropología por la Pontificia Universidad Católica del Perú.

Licenciada en Ciencias de la Comunicación por la Universidad de Lima.
\end{abstract}


Keywords: environment, ecology, theoretical framework, natural resources and communicator's reflection.

\section{1. ¿Las lomas son sólo para resbalarse?}

Desde hace algún tiempo queríamos saber el significado de algunos términos que hoy se escuchan por muchos lugares; y que han dado origen, no sólo a la formación de grupos e instituciones, sino que también han obligado a la empresa privada y al propio ESTADO a TOMAR un conjunto de acciones y decisiones; como también a la elaboración de propuestas; hoy en ejecución.

Nos referimos a términos como CONSERVACIÓN, MEDIO AMBIENTE, ECOLOGÍA, TERRITORIO, ETC.

Casi al azar, nos descubrimos paseando con Andrés Alencastre en las "Lomas de Pachacámac", lugar cercano a Lima-ciudad; que hasta ese día significaba para nosotros, sólo el lugar de los restaurantes "campestres", de venta de chicharrones -obligados después de fiestas y amanecidas-. $\mathrm{Y}$ por supuesto, como peruanos que somos, también sabemos que fue un gran santuario. De qué época? No lo recordamos. Las palabras se nos mezclan al querer recordar: fue en la época preincaica?, no: fue durante el imperio incaico. Esta confusión es porque nuestro gran repertorio cultural, que nos deja como herencia los 12 ańos de "Servicio educativo obligatorio", que debemos pasar; porque "el que estudia triunfa", no nos permite, reconocer ni nuestros territorios cercanos, sus características naturales, menos aún a sus pobladores.

Desde nuestra ignorancia, que se explicita quizás en el uso indebido de términos, nos animamos a preguntarle a Andrés -PINKY-para sus conocidos, el por qué de su interés en las lomas; y su preocupación por lo que él llama la RECUPERACIÓN DEL CAPITAL FÍSICO Y SOCIAL. Proyecto al que ha dado, hasta la fecha, 13 años de su vida. Según nos cuenta, algunos años con financiamiento, pero en otros sólo por el entusiasmo que encontró en la población. Y el terco objetivo que lo lleva a intentar la RECUPERACIÓN DE TERRITORIOS, hasta hoy, usando sus propias palabras INVISIBILIZADOS. 
Le preguntamos cómo se generó este interés. Y la respuesta nos sorprende, escuchando una exposición que desde una perspectiva histórica, pretende descubrir; mejor dicho, EXPLICITAR el USO DE LOS TERRITORIOS.

El Perú, para él, es un país muy rico, pero desconocido, poco estudiado; por ende, desaprovechado. Más aún en el contexto actual, en el que el liberalismo económico no encuentra sentido ni al estudio histórico de su capital y/o recursos naturales; y que elabora políticas de planificación urbana y rural, desconociendo -casi por completo- LOS USOS DE LOS TERRITORIOS.

Le preguntamos tímidamente, por qué le fue necesario "viajar" al pasado. Y nos responde, REFIRIÉNDOSE A LA ÉPOCA PREHISPÁNICA "antes el uso de los territorios tenía un SOPORTE EN UNA ORGANIZACIÓN SOCIO-POLÍTICA, PERO EN LA ACTUALIDAD ES UN REFLEJO DE LA ECONOMÍA GLOBALIZADA"

Pero, insistimos "¿Qué tiene que ver la idea de RECUPERACIÓN DE TERRITORIOS CON EL DESARROLLO DEL PAÍS?”. Andrés, categóricamente nos responde que LA RECUPERACIÓN Y EL USO ADECUADO DE ÉSTOS, constituye para él, OTRA ENTRADA para luchar por liquidar, disminuir el estado de pobreza, de iniquidad en el que se encuentran la mayoría de peruanos"

La respuesta inteligente sorprende nuestra ignorancia; pues habíamos escuchado y entendíamos que la CONSERVACIÓN, los RECURSOS NATURALES, el MEDIO AMBIENTE, tenían que cuidarse. Tímidamente las razones argumentaban "porque se acaba", "porque es parte de nuestro patrimonio". Pero las campañas de propaganda, al menos para nosotros, han apuntado -tácitamente-, más que se tenga una ACTITUD DE RESPETO HACIA LA NATURALEZA, que a decirnos en qué consiste la "naturaleza"; y qué son, por fin, los llamados "recursos naturales".

Yo por lo menos, sentía, cuando escuchaba algunos de los spots que las grandes campañas sobre medio ambiente han divulgado, que debía portarme ante la naturaleza como ante un ser humano. Es decir, confieso que asociaba el discurso sobre la "naturaleza", a una suerte de "Declaración de los derechos humanos", pero en versión "verde". Lo que 
me posibilitó aprender a no pisar los jardines, a no arrancar flores -así fuera una sola-, y a intentar aprenderme el nombre de árboles, plantas. Eso sí, insectos, no.

Por ello, cuando visité el proyecto en el que trabaja Pinky, y descubrí que los cerros eran ¡VERDES! Perdón, estaban cubiertos-completamentede verdor. ¿ASI SE DICE? No pude menos que reconocer, que lo único que pude experimentar fue un sentimiento, casi bucólico, por estar en el ¡“campo”! Sin embargo, no sabía, qué los hacía tener verdor; si acabábamos de pasar por el lado -tierra- de los mismos cerros. Perdón -lomas-.

Tímidamente, y con preguntas vagas, pude enterarme; es decir, informarme de "los aspectos técnicos" que nadie nos explica. Aquellos que tienen que ver con el conocimiento profundo de los USOS DEL TERRITORIO, las características climáticas, la influencia del mar sobre estas estribaciones de nuestra cordillera, etc.

Prefiero dejar al propio PINKY estas explicaciones, que la frase "aspectos técnicos" permite dejarlas de lado. Yo termino, contando que no tuve necesidad de seguir preguntando y exhibiendo mi ignorancia, a quien ha pasado 13 años de su vida en la tarea de RECUPERACIÓN DE TERRITORIOS; porque fueron los propios pobladores quienes con un admirable conocimiento de su entorno, su pasado, sus potencialidades, etc. ; me iban contando -como si fuera un cuento-

La historia de su TERRITORIO, sus características climáticas, el por qué de sus variabilidades. Mientras me mostraban orgullosos, las mejoras que habían logrado en lo que - pobremente- llamamos plantas. Todo ello, gracias al saber adquirido y a la APROPIACIÓN QUE DE ESTE HAN REALIZADO A LO LARGO DE 13 AÑOS DE ESFUERZO, DE DUDAS, DE DISCUSIONES; es decir, de trabajo. Que ahora muestran con orgullo y profundo conocimiento de lo que tienen, de lo que saben.

TERMINO CON UNA FRASE QUE LE ESCUCHÉ A PINKY, "Conservar y usar de manera sostenible".

\section{A propósito del medio ambiente}

El texto que presentamos a continuación es producto de una conversación que sostuvimos con uno de los ingenieros agrónomos 
que trabaja hace años con la población. El interés tenía que ver con uno de los temas más importantes en la agenda del DESARROLLO a nivel mundial: "LA CONSERVACIÓN Y/O RECUPERACIÓN DEL MEDIO AMBIENTE”. Si bien la preocupación por el cuidado, respeto y preservación del medio ambiente, se constituye como uno de los campos en los que se advierte el surgimiento de numerosos proyectos, cuyo objetivo es la CONSERVACIÓN DEL CAPITAL NATURAL; el tema no es nuevo.

Aunque no lo parezca, la relación hombre/naturaleza ha sido, como hasta ahora, un motivo de reflexión en diferentes disciplinas. Sin embargo, es la filosofía, la disciplina en la que se suele ubicar esta vieja temática. Nuestra propuesta conceptual, a diferencia de la mayoría, no se centra únicamente en el "elemento" naturaleza, sino que, partiendo de una mirada holística, incluye al hombre como otro "elemento" que forma parte de eso que se llama tan fácilmente "naturaleza".

Se trata del peruano Andrés Alencastre, profesional que desarrolla y ejecuta proyectos, genéricamente llamados como PROTECCION DEL MEDIO AMBIENTE, o similares; viene trabajando desde hace trece años - CON LA PROPIA POBLACIÓN- UN PROYECTO DE RECUPERACIÓN FÍSICO-SOCIAL de lo que él llama PAISAJE SOCIAL. Lo interesante del planteamiento del proyecto es que se ha logrado "construir" un marco conceptual sólido, que coge elementos de diversas disciplinas, como la historia, la geografía, la antropología, etc. Lo que nos parece importantísimo es el aporte que brinda esta experiencia con esta suerte de "marco teórico", casi inexistente en proyectos similares. Lamentablemente, se cree que la producción de conocimiento, es tarea de las ciencias sociales únicamente.

Queremos resaltar este aspecto, pues por lo general los profesionales que no proceden del campo que equivocadamente se designa como "LETRAS", no son conscientes que las actividades de "producción", o el aprendizaje de aspectos "técnicos"; son de carácter PRÁCTICO. Lo cual hace que ni se imaginen que puedan darse procesos de producción de conocimiento, a partir de lo que vienen realizando.

A nuestro entender, lo valioso de la propuesta es que a través de sus 13 años de dedicación (con o sin financiación) han logrado la RECUPERACIÓN DE LAS LOMAS DE PACHACAMAC, y 
CONSTRUIR UN MARCO CONCEPTUAL, que podría ser de gran utilidad para los proyectos que en esa línea se vienen desarrollando a nivel mundial.

La propia metodología de TRABAJO, que se implementó fue, partir del trabajo CON LOS PROPIOS POBLADORES, EN EL PROYECTO MENCIONADO. LOS PROPIOS POBLADORES, manejan un capital de INFORMACIÓN SOBRE SU ZONA, (territorio para ellos), que expresa la asimilación, al interior del proyecto y la metodología, la difusión de un conjunto de saberes que el POBLADOR SE HA APROPIADO, a partir de la peculiaridad del trabajo del proyecto.

Esto último, constituye, para comunicadores como nosotros un LOGRO IMPORTANTÍSIMO que demuestra una vez más, la importancia que tiene la reflexión sobre el CONOCIMIENTO, las formas de APRENDIZAJE; y la acumulación no de INFORMACIÓN, sino de saberes que ya son parte del CAPITAL CULTURAL DEL POBLADOR. Este hecho, quizás, poco visible para quienes esperan objetivos de tipo más "práctico", es para los comunicadores la PRINCIPAL MISIÓN que los PROYECTOS DE PROMOCIÓN Y DESARROLLO DEMANDAN. Pues, los procesos de construcción de conocimiento, dependen no sólo del "CONVENCIMIENTO" que el trabajador debe hacer en la población; sino de las peculiares gramáticas de percepción y códigos de recepción, que constituyen la matriz cultural desde la que el poblador "mira" y "aprehende" la realidad.

En ese sentido, el presente texto, se proponía PRESENTAR EL MARCO TÉORICO que sobre "EL PAISAJE SOCIAL Y SU RECUPERACIÓN", sustenta esta experiencia, en el que los profesionales no sólo han hecho labores de "acompañamiento" al proyecto; sino de un proceso conjunto de procesos y aprendizajes, al interior de la población.

\section{La ecología humana, es útil?}

La enseñanza de la Ecología es un tema que cada día toma más importancia. Por tanto, es menester incorporarlo a la formación universitaria. Más aún de quienes tienen la tarea de dar a conocer o 
difundir el por qué de su importancia. Es importante mencionar algunos aspectos de la "Ecología Humana" como: La Historia de la Ecología, La Contaminación, El Efecto Invernadero, etc., ya que muchos estudiantes desconocen estos conceptos y por lo tanto, también, los efectos que pueden causar sobre la vida de nuestro planeta. Para entender lo que significa la "Ecología Humana", empecemos recordando la dolorosa visión del guanay agonizando en las turbias aguas de una playa limeña por un irresponsable derrame de petróleo, poco o nada podían hacer por el ave desfalleciente. Desde entonces nos sentimos impotentes, indignados del poco respeto que se le prodiga a la vida. Recién ahí puede entenderse la inmensa calidad humana de los grandes ecologistas, los defensores del medio ambiente, y profesionales diversos, pues comprenden y se preocupan por los habitantes y todos los seres vivos que habitamos la tierra.

\subsection{Concepto}

La Ecología Humana, es el estudio de las relaciones entre los seres humanos y su entorno. Su objetivo principal es investigar el modo en que los seres humanos adaptan su genética, fisiología, cultura y conducta al medio físico y social. También podemos definir la ecología humana como el estudio científico de las relaciones, en tiempo y espacio, entre la especie humana (Homo sapiens) y otros componentes y procesos de los ecosistemas de los cuales forma parte.

\subsection{Objetivo del estudio de la ecología humana}

El objetivo principal del estudio de la ecología humana es conocer la forma en que las sociedades humanas conciben, usan y afectan el ambiente incluyendo sus respuestas a cambios en tal ambiente, a los niveles biológicos, sociales y culturales. En la actualidad la ecología humana se incluye dentro de un amplio marco ecológico y evolutivo, e incluye el estudio del impacto humano sobre el entorno, la nutrición, los desastres ecológicos y la demografía. Aunque el alcance de la ecología humana es inmenso, existe un aspecto común que es la comprensión del modo en que los seres humanos responden a su entorno, sea éste 
genético, fisiológico, de conducta o de cultura. Si el planeta tierra se hubiera formado un primero de enero, la especie humana habría tenido que esperar hasta el día 31, a las once de la noche, para aparecer. Sin embargo, a pesar de su breve estancia sobre el planeta, el hombre ha invadido todos los rincones del globo y los ha ido modificando con la finalidad de satisfacer sus necesidades más inmediatas. La Ecología humana desde una perspectiva teórica e histórica breve es la tercera perspectiva relacionada con la sociología, además de la antropología cultural y la psicología social, y estudia las conexiones de la población con el ecosistema y este es su tema importante. Es en sí mismo como un método por el enfoque de la selección que hace de sus contenidos como díadas o como tríadas: la 'población' adaptada por la cultura y el espacio, - que se compone de 'organización social' y 'tecnología' -, a su 'medio ambiente' para sobrevivir. El ecosistema formado por estas cuatro variables resulta inestable y en el límite de este proceso en conflicto está el planteamiento ecológico de adaptación, porque si la población se expande el medio ambiente se deteriora. $\mathrm{O}$ no responde a las nuevas necesidades, entra entonces en conflicto con el diseño que habíamos deseado para la comunidad humana. Ejemplo son los problemas de tráfico en las ciudades. Park y Burgess en 1920, desde la ecología vegetal y animal y desde Simmel, introducen el término y se crea la Escuela de Chicago, que desarrolla la ecología urbana y que sigue vigente en la parte introductoria de los programas académicos. El Dpto. Sociología II (Ecología Humana y Población) de la Facultad de Sociología de la UCM contiene programas y bibliografía. La Ecología Humana es más popular en América como ecología urbana y ecología regional, que en Europa.

\section{El impacto ambiental}

El impacto ambiental es un choque causado por el hombre en desmedro del medio ambiente. Estos impactos también pueden ser causados por fenómenos naturales, como los choques de meteoros, pero la acción humana también ha llegado a destruir plantas y animales. Muchas especies animales y vegetales se han extinguido en varios países, 
por la caza indiscriminada, por la sustitución de bosques por cultivos, plantaciones y urbanizaciones, por la introducción de productos químicos como el DDT que viaja grandes distancias en tiempo seco y al que solo los insectos se han vuelto resistentes. Históricamente, el hombre ha considerado la atmósfera y los océanos como dos depósitos de capacidad infinita, donde es posible abocar toda clase de desechos. El dióxido de carbono que los procesos industriales acumulan en la atmósfera, a un ritmo acelerado, intercepta las radiaciones caloríficas de la superficie terrestre y las devuelve a ella, trayendo como consecuencia un ascenso paulatino de la temperatura global, que conocemos con el nombre de efecto invernadero. Los gases emitidos junto con las partículas sólidas, como polvo y hollín, se combinan con el vapor de agua de la atmósfera y producen un aumento de la cubierta de nubes; estas nubes son más estables cuanta mayor altura alcanzan. Las consecuencias sobre la modificación del clima afectarán a toda la biosfera y la preocupación por el tema debe ser compartida por todos los países de la tierra. La emigración de las gentes del campo a las ciudades y del interior a las costas ha originado que muchas sustancias procedentes de ecosistemas distintas, se concentren en las ciudades y se acumulen en sus alrededores en forma de desparramados humanos. Debemos aprender de los pueblos indígenas, que conocen su medio desde milenios y saben como utilizarlo sin causar daño. Ejemplo, los nativos amazónicos usan la tierra por 4 años y luego se mudan, entonces la floresta se restituye en poco tiempo; los colonos adaptados al medio colonizado tienen otra conducta, utilizan todos los bosques, causando grandes problemas a la reconstitución de los suelos y de la vida. Son los indígenas en el mundo los que aún conservan muchos ecosistemas de gran valor para la humanidad, porque son poseedores de conocimientos y técnicas tradicionales para cuidar su medio. Estos pueblos a veces son amenazados con la pérdida de su diversidad biológica y cultural por fuerzas externas y por la inacción de los gobiernos nacionales. Ejemplo los nativos de la selva, en el Perú, están siendo amenazados nuevamente por el brote del terrorismo, están solicitando apoyo al gobierno central y ellos no hacen nada para impedirlo. Los pueblos indígenas antes y ahora contribuyen con la diversidad biológica en sus países y el mundo, es pues tarea muy importante velar por su existencia. La conservación 
de los recursos naturales consiste en el empleo prudente de los recursos renovables que son esenciales para la supervivencia humana. El hombre como depositario de los recursos de las generaciones futuras está obligado a administrarlos cuidadosamente, a prever y planear la conservación de todos los recursos naturales. Desde comienzos del presente siglo, han demandado el desarrollo de acciones de conservación de los recursos naturales en base a la cooperación internacional, para velar por el futuro de la propia humanidad. Pero, a pesar de los llamados de atención de los especialistas y conservacionistas y de los organismos internacionales, la destrucción de los hábitats crece día a día. La conservación es tarea de todos. Es tarea esencial de los gobiernos y los individuos, es urgente una educación masiva en temas ecológicos, la promulgación de leyes que regulen la utilización de los recursos, el fomento de la investigación básica que permiten conocer los recursos, especialmente los que están en peligro de extinción. 